\title{
La Ciencia Política en Colombia: una \\ disciplina en proceso de \\ institucionalización
}

\author{
Political Science in Colombia: a \\ discipline in process of \\ institutionalization
}

\section{Santiago Leyva Botero \\ María Fernanda Ramírez \\ Patricia Muñoz Yi \\ Andrés Preciado \\ José Antonio Fortou}

\section{Resumen}

Este artículo explora algunos de los avances en la institucionalización de la Ciencia Política en Colombia, teniendo en cuenta que ya se superaron las etapas de su creación como vocación (19681990) y de su expansión como profesión (1990-2006). Para ello, realiza un breve recorrido por la historia de la Ciencia Política en el país, haciendo énfasis en la segunda mitad del siglo XX, a la vez que da cuenta de las distintas evaluaciones que se han hecho del estado de la disciplina de manera retro y prospectiva. Así mismo, muestra cómo los avances en el proceso de institucionalización disciplinar se pueden identificar en el marco de la creación de la Asociación Colombiana de Ciencia Política (ACCPOL) y el establecimiento de contenidos comunes en los programas de formación politológica existentes en el país.

\section{Palabras clave}

Ciencia Política en Colombia; Institucionalización de la Ciencia Política; Asociación Colombiana de Ciencia Política.

\section{Abstract}

This paper explores some of the advances in the institutionalization of political science in Colombia, considering that the stages of its creation as a vocation (1968-1990) and its expansion as a profession (1990-2006) have been completed. To do this, the article presents a brief overview of the history of political science in the country, with emphasis on the second half of the twentieth century. It also shows that advances in the institutionalization process of the discipline are linked to the creation of the Colombian Association of Political Science (ACCPOL) and the establishment of shared content and criteria in political science programs within the country.

\section{Keywords}

Political Science in Colombia; Institutionalization of Political Science; Colombian Association of Political Science. 


\section{Introducción ${ }^{1}$}

Este artículo explora algunos de los avances en la institucionalización de la Ciencia Política en Colombia, teniendo en cuenta que ya se superaron las etapas de su creación como vocación (1968-1990) y de su expansión como profesión (1990-2006). En este sentido, se propone que a partir de nuevas tendencias como la creación de la Asociación Colombiana de Ciencia Política (ACCPOL), la continuidad relativa de los congresos y encuentros nacionales, el surgimiento de programas de formación de doctorado y la consolidación de varias revistas colombianas en el escenario internacional, es posible hablar hoy del surgimiento de una nueva etapa de institucionalización de la Ciencia Política colombiana. Estos cambios, en algunos aspectos aún incipientes y en otros ya bastante consolidados, transforman el escenario en comparación con el momento en que se produjeron la primera y segunda generación de trabajos que iniciaron el debate disciplinar (LEAL BUITRAGO, 1988; SÁNCHEZ, 1994; MURILLO CASTAÑO y UNGAR BLEIER, 1999; LOSADA, 2004; BEJARANO y WILLS, 2005; CUÉLLAR ARGOTE, 2007).

En Colombia la creación de la disciplina despegó a nivel nacional apenas en la década de los noventa, después de existir desde 1968 (por más de veinte años) en un solo pregrado y en un par de maestrías creadas desde los años setenta. Desde entonces, y especialmente desde 1996, se produjo un crecimiento muy acelerado de la formación en la disciplina de la Ciencia Política en todos los niveles de estudios (DUQUE DAZA, 2013; FORTOU, LEYVA BOTERO, PRECIADO y RAMÍREZ, 2013). Esta explosión, tal y como señala Altman (2006) para otros países de América Latina, se realizó en medio de dinámicas de mercado, con una oferta mayoritaria de universidades privadas bajo una muy débil regulación y generalmente bajo una fragmentación importante de propósitos y diseños académicos. Se trató en Colombia de un proceso que "explotó" en muy poco tiempo: pasó de dos programas de pregrado en 1993 a 32 en el 2011, lo que indudablemente implicó que el proceso de expansión no se dio de manera orgánica. Esta característica llevó a que la planta profesoral de la mayoría de los nuevos programas en Colombia no fuera formada en una disciplina común, y a que tampoco contara, durante estos años de expansión, con un proyecto colectivo para la creación y autonomización de una nueva disciplina. Adicionalmente, el momento de la "explosión" de la disciplina en Colombia coincidió también con la

\footnotetext{
${ }^{1}$ Este artículo es producto de la investigación "Institucionalización de la Ciencia Política en Colombia" desarrollada en el 2012 en la Universidad EAFIT para preparar académicamente el evento Primer Encuentro Nacional de Facultades y Programas de Ciencia Política organizado por la Asociación Colombiana de Ciencia Política (ACCPOL), la Universidad EAFIT y la Universidad Pontificia Bolivariana el 8 y 9 de Noviembre en Medellín, Colombia. Este artículo fue posible gracias al convenio de especial de cooperación 0658-2012 entre la Universidad EAFIT y el Departamento Administrativo de Ciencia Tecnología e Investigación - Colciencias de la República de Colombia. Este artículo fue pregrado en el marco del grupo Estudios sobre Política y Lenguaje del Departamento de Humanidades de la Universidad EAFIT (cat. A1-Colciencias).
} 
crisis y fragmentación de la Ciencia Política a nivel internacional (SARTORI, 2004; CANSINO, 2008). Es necesario recordar que después de la crisis del conductismo, la Ciencia Política de los años noventa estuvo dominada por una visión pesimista sobre las posibilidades de tener un objeto común entre los cultores de la disciplina que les permitiera tener una identidad y un propósito común (ALMOND, 1999). Al contrario de otras áreas, se trataba entonces de una disciplina que no contaba con un método privilegiado, y que por la polisemia misma de su objeto parecía dispersarse en una multiplicidad de intereses y focos que hacían imposible un diálogo entre sus propios practicantes (ALMOND, 1999).

Todo lo anterior implica que, en el momento de su expansión como profesión universitaria, la Ciencia Política colombiana enfrentó un doble reto, aquel creado por su explosión y también el dado por la fragmentación de sus cultores en varios campos percibidos como irreconciliables. Ambos fenómenos crearon una serie de divisiones locales que hoy se constituyen como un desafío a superar.

Entre otras divisiones, el saber disciplinar resultaba fraccionado entre los enfoques cuantitativos que enfatizan su causalidad y los cualitativos que destacan su polisemia, entre los enfoques científicos y los que tratan de rescatar los 25 siglos de historia de la teoría (filosofía) política, y entre los enfoques que tenían un diálogo más abierto con las ciencias sociales (estudios políticos) y la "Ciencia Política" con una visión más autónoma y disciplinar. Todas estas separaciones implicaron que, no existió en Colombia un acuerdo sobre qué se debía tener en cuenta a la hora de diseñar los programas de Ciencia Política, hacia dónde se debía dirigir la investigación y qué se debía leer para aprender de la disciplina.

En este trabajo, se pretende ilustrar que la institucionalización de la disciplina se puede llevar a cabo mediante el fortalecimiento institucional en múltiples niveles, entre otros, la consolidación de la asociación de politólogos (ACCPOL) y el establecimiento de competencias y contenidos comunes que permitan mejorar el nivel de formación de sus profesionales. Para cumplir con tal propósito el artículo, luego de esta introducción, inicia con un recorrido por la historia de la Ciencia Política en Colombia, haciendo énfasis en la segunda mitad del siglo XX; seguidamente, da cuenta de las distintas evaluaciones que se han hecho del estado de la disciplina de manera retro y prospectiva. En el tercer apartado, se aborda el proceso de institucionalización en el país con una mirada a la Asociación Colombiana de Ciencia Política - ACCPOL. Una vez revisada la historia y los debates de institucionalización, la cuarta parte del artículo hace una descripción y análisis de macrocurrículos de Ciencia Política en Colombia, tratando de evidenciar puntos de encuentro y diferencias en las distintas apuestas institucionales para, finalmente, presentar algunas conclusiones relativas a los avances y los retos en la institucionalización. 


\section{Un recorrido por la historia de la ciencia política en el país}

Este apartado se propone un recorrido por la historia de la Ciencia Política en el país, particularmente a partir de la segunda mitad del siglo XX. Para esto, se revisan y sintetizan las principales "narrativas" de la historia disciplinar.

El primer departamento de Ciencia Política que se creó en el país fue el de la Universidad de los Andes en 1968. Su nacimiento se dio en un ambiente de polarización producido por una división entre los paradigmas marxistas y el estructuralismo funcional (BEJARANO y WILLS, 2005). Esta división teórica y política de la época trasladaba el desencuentro a las mismas instituciones educativas, donde la polarización y el "conflicto" afectaron principalmente a las facultades de Sociología, muchas de las cuales llegaron a ser cerradas por sus problemas internos.

La creación de este Departamento debe enmarcarse dentro del proceso más amplio del surgimiento de las ciencias sociales en la academia colombiana, así como del contexto sociopolítico. El proceso modernizador laico del Frente Nacional a partir de 1958 estuvo mediado por una gran expansión del cuerpo estudiantil y por la inclusión de las clases medias en la educación superior. Dentro de este contexto, el surgimiento de la Ciencia Política en Colombia buscaba propiciar una clara separación entre militancia e ideología y, por lo tanto, imprimirle al estudio de la política una "naturaleza secular" que permitiera "una aproximación más moderna, racional y por sobre todo no-confesional a los problemas y fines de la actividad política" (BEJARANO y WILLS, 2005, p. 113).

Los primeros trabajos en Ciencia Política en Colombia poseen visos conductistas, orientados por la Ciencia Política estadounidense. Las primeras investigaciones realizadas allí muestran una cierta ruptura con temas de Filosofía (política), Derecho (constitucional) y Sociología que habían dominado el campo de los estudios políticos en Colombia. Este rompimiento puede verse en el surgimiento de un nuevo orden temático donde sobresalen temas como el "comportamiento legislativo, partidos políticos y grupos de presión, elecciones y participación electoral” (BEJARANO y WILLS, 2005, p. 113-114). Los primeros trabajos se caracterizaban por la utilización de métodos sofisticados para la época, como la realización de encuestas a ciudadanos y congresistas, para así "entender cómo las diferentes características de los individuos afectan su comportamiento político" (BOTERO, 2011, p. 22). El conductismo que irrumpe en la Ciencia Política en Colombia trata entonces de romper con el evolucionismo (uso de la historia) y también con el uso de la filosofía normativa en el estudio de la Ciencia Política.

Desde otra perspectiva, Murillo y Ungar $(1999$, p. 2) señalan que para entender el origen de los estudios políticos ${ }^{2}$ también es necesario mirar cómo la

\footnotetext{
${ }^{2}$ Nótese que no se habla de ciencia o ciencias. La discusión sobre la denominación de la disciplina - ciencia, ciencias, estudios - es larga y amerita un estudio aparte. Sin embargo, se hará referencia ocasional y lateral a dicha diferenciación.
} 
Sociología Política de la época empieza a tratar ciertos temas: "violencia, procesos de urbanización, movimientos estudiantiles, migración rural-urbana, movilidad social, estructuras familiares y tenencia de la tierra, entre otros". Sin embargo, estos estudios se dieron dentro de una academia cada vez más polarizada, que mezclaba la militancia ideológica con ciertas características antisistema, que llevaron finalmente al cierre de las facultades de Sociología de la Pontificia Universidad Javeriana y de la Universidad Nacional a principios de los setenta (MURILLO CASTAÑO y UNGAR BLEIER, 1999).

En 1986, casi 20 años después de la génesis uniandina, se crea en la Universidad Nacional de Colombia, sede Bogotá, el Instituto de Estudios Políticos y Relaciones Internacionales (IEPRI) con un corte diferente al de la Universidad de los Andes. Murillo y Ungar (1999) resaltan cómo la emergencia del IEPRI en el momento más caldeado de la violencia de los años ochenta le da una mayor orientación hacia un "corte interdisciplinario". Bejarano y Wills (2005), por su parte, señalan que estas diferencias de orientaciones se deben, en parte, a que el IEPRI se creó bajo una mayor influencia de la academia europea y, por lo tanto, con un enfoque más amplio, que no fetichiza las Ciencias Políticas en su separación de las ciencias sociales y que no exagera la independencia de la política de otros aspectos de la sociedad. Ambas razones llevaron a este nuevo instituto a trabajar desde un principio con una perspectiva más interdisciplinaria, centrada en las articulaciones entre Estado, régimen político y violencia.

En 1987, el gobierno nacional creó la Comisión sobre Violencia y Democracia, liderada por Gonzalo Sánchez del IEPRI. La publicación que resultó del trabajo de la Comisión - el libro Colombia: Violencia y Democracia - puso de relieve la discusión sobre las "causas objetivas" de la violencia. Durante esta década, aparece además el clientelismo como tema, el estudio del comportamiento electoral (desde un enfoque psicosocial), el comportamiento partidista y la tesis del "cierre del sistema" durante el Frente Nacional (FN) y la crisis del bipartidismo (partidos tradicionales divididos en fracciones y facciones) con el desmonte del mismo (BOTERO, 2011).

Durante la siguiente década (1990-2000), la hiperfragmentación del sistema de partidos copó la atención de los politólogos colombianos interesados en temas electorales y partidistas (BOTERO, 2011). No obstante, el clientelismo no pasa de moda y sus mutaciones (hacia lo que Andrés Dávila Ladrón de Guevara llama un "clientelismo de mercado") han hecho que siga siendo un tema estudiado. El narcotráfico, la guerra contra las drogas, el Plan Colombia y el conflicto armado ocuparon las páginas de los trabajos sobre violencia y seguridad. Así mismo, el contexto global tras la Guerra Fría y el papel de Colombia en este nuevo escenario fue tema recurrente. Finalmente, el debate a la Constitución Política de 1991 - en su génesis, pero también las evaluaciones de sus efectos hizo presencia en la Ciencia Política nacional. 
Ya en el nuevo siglo, la Ciencia Política colombiana ha seguido ampliando el espectro de temas tratados. Por ejemplo, los estudios sobre partidos y elecciones se han enfocado en las causas y los efectos de las reformas políticas especialmente la de 2003 -, en las relaciones entre violencia, criminalidad, democracia y representación, y la realización de encuestas de opinión como la del Latin America Public Opinion Project (LAPOP). Tras la desmovilización paramilitar, ha habido una renovada discusión sobre los procesos de desarme, desmovilización y reintegración y de justicia transicional. Así mismo, la reelección como figura política y sus implicaciones en el sistema político han sido objeto de numerosas publicaciones. Algunas de estas se relacionan, obviamente, con las discusiones en torno a la Constitución del 91, la cual ha sido a su vez objeto de varios trabajos, especialmente en el contexto de sus 20 años. Igualmente, los estudios sobre violencia, conflicto, orden y seguridad no han perdido su importancia en la Ciencia Política colombiana ${ }^{3}$.

Finalmente, Bejarano y Wills sostienen que las preocupaciones de la Ciencia Política hoy se distancian de las de anteriores épocas en el siguiente aspecto:

A los temas tradicionales se han venido a sumar nuevas preocupaciones: movimientos sociales, sociedad civil, ciudadanía, formación de la esfera pública, descentralización, proceso constituyente, reforma del Estado y diseño institucional, resolución pacífica de conflictos y justicia transicional (BEJARANO y WILLS, 2005, p. 119).

Pero quizás uno de los desarrollos más importantes para la disciplina en el país fue la "explosión" de programas de pregrado y posgrado en Ciencia Política y afines a partir de la década de 1990. Este proceso se relacionaría con la descentralización que llevó la necesidad del quehacer político-práctico a las regiones y la expansión de los regímenes ejecutivos en la nación. La Gráfica 1 muestra dicha explosión en los programas de pregrado en Ciencia Política en el país (un total de 33 a octubre de 2012), haciendo énfasis en su carácter regional.

\footnotetext{
${ }^{3}$ Para un balance de los estudios sobre conflicto armado en Colombia, ver Leal Buitrago (2008).
} 
Gráfica 1 - Programas de Ciencia Política en Bogotá comparado con programas en otras regiones del país, 1969 - 2012

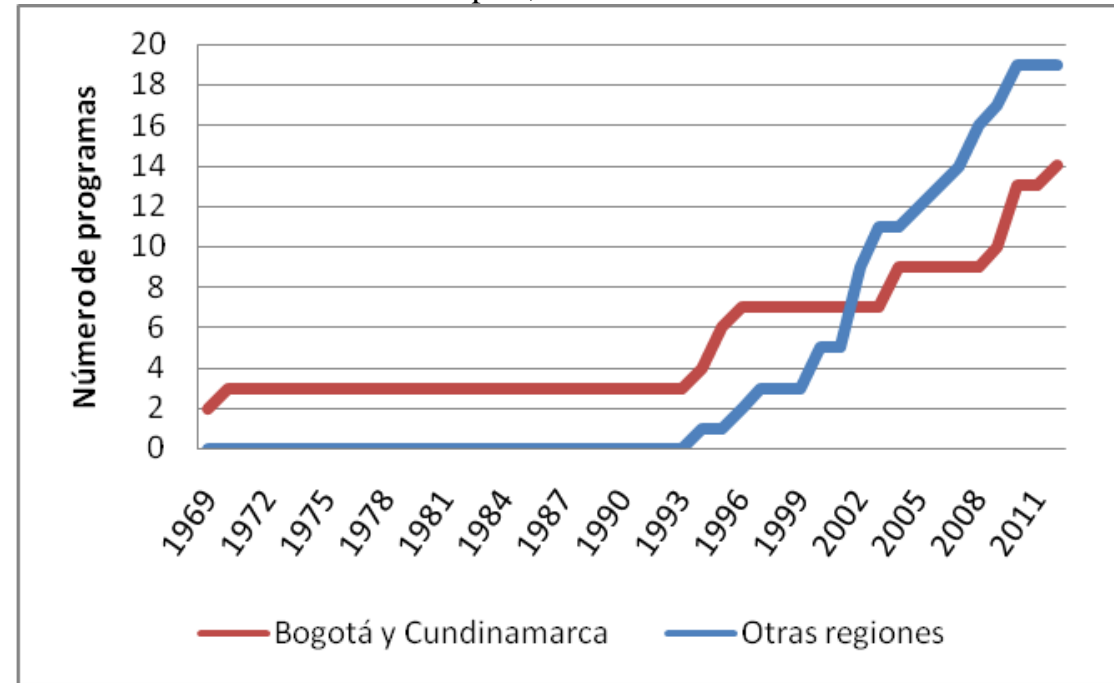

Fuente: Elaboración propia con base en datos del Sistema Nacional de Información sobre la Educación Superior (SNIES).

Si bien es posible criticar la proliferación de programas en la disciplina desde una perspectiva escéptica y orientada hacia el cuestionamiento de la calidad de los mismos (LOSADA, 2004), ciertamente este aumento cuantitativo constituye evidencia de un marcado interés en el país por pensar la política desde la academia y formar politólogos a nivel profesional (CUÉLLAR ARGOTE, 2007).

Este resumen de los principales hitos sirve para trazar una sintética historia de la disciplina. Dicha historia empezaría con la profesionalización lenta de las ciencias sociales en Colombia a partir de la década de 1950; seguiría con el establecimiento de la primera carrera de Ciencia Política en el país, la de la Universidad de los Andes, en 1968; pasaría por la ampliación del espectro de temas tratados por politólogos y estudiosos de la política colombiana en las tres décadas siguientes; y desembocaría en una explosión sin precedentes de programas de formación profesional - en pregrado y posgrado - en Ciencia Política, con especial énfasis en la expansión de esta disciplina hacia las regiones.

\section{Contiendas sobre el estado de la disciplina en Colombia}

Con esta historia en mente, es pertinente pasar revista a las distintas evaluaciones que se han hecho de la disciplina en los últimos años. En otras palabras, este apartado propone un barrido general y sintético de la literatura preocupada por el estado del arte de la Ciencia Política en Colombia: su desarrollo, su presente y sus perspectivas.

Numerosos estudiosos han intentado apuntalar "en qué va" la disciplina hoy en el país. De manera similar a lo que sucede en el plano internacional, 
encontramos perspectivas encontradas que podemos clasificar en dos grupos: las visiones optimistas por un lado, y otras mucho más escépticas o críticas frente a la actualidad del proceso de profesionalización y ampliación de la Ciencia Política colombiana. La Tabla 1 resume algunas de las principales posiciones en el debate.

Tabla 1 - Visiones sobre el estado de la disciplina en Colombia

\begin{tabular}{c|c}
\hline Optimistas/positivas & Escépticas/críticas \\
\hline "Profesionalización" & "Proceso en marcha" (MURILLO \\
(LEAL BUITRAGO, 2011) & CASTAÑO y UNGAR BLEIER, 1999) \\
\hline "Reconocimiento" & "Crisis" (ALONSO ESPINAL, 2001) \\
\hline (SÁNCHEZ DAVID, 2001) & "Preocupante proliferación"; "Está de \\
me vocación a disciplina" (BEJARANO y & moda" (LOSADA, 2004) \\
\hline WILLS, 2005) & "Retorno a la disciplinariedad" \\
\hline “Consolidación" (CUÉLLAR ARGOTE, & (RESTREPO, LÓPEZ, LÓPEZ y \\
2007) & SÁNCHEZ, 2008) \\
\hline
\end{tabular}

Fuente: Elaboración propia.

El campo de las visiones optimistas sobre el estado de la Ciencia Política en Colombia se ve potenciado por narraciones de su historia como la que ofrecen Bejarano y Wills (2005) y Leal Buitrago (1988; 2011). En ambos casos, el desarrollo de la disciplina ha sido un proceso lento y arduo, casi épico, que comenzó con unos pocos estudiosos formados en el exterior y algunos profesores visitantes y que, cuatro décadas después, se ha caracterizado por una creciente proliferación y una clara expansión de sus enfoques, métodos y temas. Para Sánchez David (SÁNCHEZ DAVID, 2001, p. 30) el tránsito ha sido claro: de los politólogos como "seres extraños cuya actividad se confundía con la de los políticos y con las miserias del poder", al reconocimiento por parte de la academia y la sociedad colombianas.

Trabajos como el de Cuéllar Argote (2007) ligan muy bien con esta perspectiva. La Ciencia Política se habría consolidado de forma "[...] si bien lenta y desfasada, [...] no sólo firme sino constante" (CUÉLLAR ARGOTE, 2007, p. 270). El elemento clave a celebrar sería la "explosión de programas" (de 2 a 33 en menos de veinte años), pues constituye una oportunidad para producir conocimiento sobre los problemas políticos colombianos (CUÉLLAR ARGOTE, 2007, p. 282). Murillo y Ungar (1999) intentan matizar estas evaluaciones. Si bien apoyan la "interpretación estándar" del desarrollo de la disciplina en el país, son cautos al caracterizar este proceso como un "proceso en marcha".

Una de las críticas más ácidas al estado de la disciplina en Colombia viene de uno de sus fundadores. Si Sartori critica a la Ciencia Política en versión estadounidense por su excesiva cuantificación y falta de verdadera metodología, Losada (LOSADA, 2004, p. 17-18, 21) critica a la colombiana por su escaso rigor 
teórico, su "clara desconexión con los principales desarrollos de la Ciencia Política contemporánea" y sus limitados intentos por realizar mediciones (cuantitativas o cualitativas) "no [la] dejan muy bien parada".

\section{La Asociación Colombiana de Ciencia Política (ACCPOL)}

En el marco de este nuevo contexto en la formación en Ciencia Política en Colombia, en el año 2008, 12 universidades fundan la Asociación Colombiana de Ciencia Política (ACCPOL) con el objetivo de servir como escenario de encuentro de todas las universidades que ofrecen programas de pregrado y posgrado en la disciplina, así como constituirse en el medio institucional de difusión de la disciplina politológica en el entorno nacional ${ }^{4}$. Este momento fundacional no es un dato menor, teniendo en cuenta lo planteado por el profesor Daniel Chasquetti (2013), esto es, que las asociaciones académico-profesionales son una consecuencia del desarrollo de la disciplina y que sus características están ligadas al nivel de institucionalización que esta haya alcanzado en el país.

La ACCPOL, en ese sentido, está concebida como un esfuerzo de construcción de comunidad académica e institucionalización disciplinar, similar al acontecido en otros países de la región como Argentina, Uruguay y Chile con asociaciones equiparables, aunque de mayor trayectoria. No obstante, una de las principales particularidades de la ACCPOL actualmente es que el grueso de las membresías es de tipo institucional, es decir, se trata de una asociación de universidades, mas no de profesionales de la Ciencia Política.

A noviembre del 2013, del total mencionado de 32 programas de Ciencia Política en el país, 23 hacen parte de la ACCPOL como miembros plenos ${ }^{5}$. Varios más se encontraban en proceso de entrada o habían mostrado interés en ingresar a la Asociación. Adicionalmente, los siete programas con Acreditación de Alta Calidad son miembros activos. El hecho de que en cuatro años la Asociación haya agrupado casi dos tercios de los programas existentes parece un avance relevante en el proceso de institucionalización disciplinar pretendido. En la ACCPOL, el centralismo académico es levemente más notable que en el país en general: 10 de los 19 programas se encuentran en Bogotá o su área de influencia inmediata (el municipio de Chía).

Dado el carácter prioritariamente institucional de la ACCPOL, tanto su asamblea de miembros como los distintos espacios que lidera (seminarios o foros) tienen como objetivo promover la discusión e investigación en torno al estado de la disciplina en el país y la forma como se enseña Ciencia Política, tanto en pregrado como posgrado. No es gratuito que busque constituirse en el epicentro de las discusiones disciplinares desde lo temático, lo pedagógico, lo investigativo, lo

\footnotetext{
4 Para más información acerca de la ACCPOL, puede consultarse su sitio web <http://accpol.org/index.html>.

${ }^{5}$ La membresía institucional de la Universidad Nacional de Colombia cobija los programas ofrecidos en las sedes de Bogotá y Medellín.
} 
macro y microcurricular. La consolidación de este tipo de esfuerzos y el logro de consensos en torno a las discusiones macrocurriculares siguen siendo perentorios en el país, máxime si se revisan contextos como el de la Comunidad Europea y el Espacio Europeo de Educación Superior (EEES), surgido a partir del Tratado de Bolonia. Por otra parte, si se toman referentes más cercanos, como las asociaciones profesionales en el Cono Sur, se puede ver que en la actualidad ocupan el centro de la escena académica (CHASQUETTI, 2013), luego de procesos paulatinos de consolidación que varían de país a país. No obstante, las discusiones temáticas y pedagógicas fomentadas desde la ACCPOL constituyen un paso importante en el fortalecimiento e institucionalización disciplinar, aún resta mucho por trabajar en los procesos de estandarización académica y cooperación profesional que propician este tipo de asociaciones en el mundo.

\section{La enseñanza de la Ciencia Política en Colombia: una aproximación desde los macrocurrículos de pregrado}

En vista del rápido crecimiento del número de programas de formación profesional en Ciencia Política a nivel de pregrado en el país ${ }^{6}$, vale la pena detenerse e indagar, por el estado de la enseñanza en esta disciplina. En este apartado se opta por un acercamiento a esta cuestión desde los macrocurrículos, es decir, a partir de los cursos o materias dictadas en los diferentes programas. La metodología adoptada para este trabajo es estadística, y se complementa con reflexiones teóricas, discusiones existentes en la literatura sobre el tema y con las declaraciones de los propios programas en sus sitios web.

Esta forma de abordar la temática permite aproximarse a una respuesta parcial a una serie de preguntas en torno a la formación disciplinar en Colombia. ¿Qué se está enseñando en Ciencia Política? ¿Cómo se está adaptando la formación disciplinar a los diferentes contextos regionales? ¿Existen apuestas novedosas o divergentes que sean expresión de identidades sub o trasdisciplinares distintas a la "ortodoxia"? ¿Hay elementos comunes en cuanto a lo que se enseña en las clases? ¿Cuáles son las tendencias generales?

Algunos trabajos ya han intentado abordar esta discusión en Colombia. Entre otros, Losada (2004), en un artículo publicado en la revista Papel Político propone una evaluación del crecimiento en el número de programas de formación y presenta un balance crítico: la Ciencia Política como disciplina estaría viéndose diluida. Pocos años después, un texto de Cuéllar Argote (2007) en la revista Civilizar aventura su propio análisis sobre el estado de estos mismos programas, la composición de sus macrocurrículos y el desempeño profesional de los

\footnotetext{
${ }^{6}$ También ha habido un crecimiento importante en el número de posgrados; ya existen en el país tres doctorados en el área (Ciencia Política en la Universidad de los Andes; Estudios Políticos y Relaciones Internacionales en la Universidad Nacional de Colombia, sede Bogotá; y Estudios Políticos en la Universidad Externado de Colombia).
} 
politólogos colombianos, entre otras cuestiones relacionadas con el problema planteado.

En aras de discutir con la visión crítica de Losada (2004) y de profundizar en algunos de los aspectos esbozados por Cuéllar Argote (2007), a continuación se presentan y discuten los resultados de un análisis estadístico exploratorio de los macrocurrículos ${ }^{7}$ pertenecientes a 28 programas de Ciencia Política en el país (del total de 32 identificados), los cuales incluyen programas dentro y fuera de la ACCPOL. En ocasiones, la estadística es complementada o contrastada con información cualitativa provista por las mismas universidades o disponible en la página web de los programas en cuestión. El objeto del ejercicio es encontrar regularidades y diferencias entre las propuestas pedagógico-académicas de los pregrados analizados $\mathrm{y}$, por esta vía, construir una suerte de "estado" de la formación disciplinar.

Los datos fueron recopilados de dos maneras: primero, se pidió a los programas que enviaran dicha información actualizada; cuando no fue posible conseguirlos por este medio, se recurrió a la información publicada en la página web. Los cinco programas no incluidos en este trabajo no enviaron información o no difunden esta información en sus páginas web. En la base de datos se incluyeron materias que todos los estudiantes de un pregrado deben cursar para acceder al título profesional y no se incluyen aquellas materias o cursos que hacen parte de lo que distintas universidades llaman núcleos de formación, ciclos básicos, complementarias, electivas, énfasis, inducción y similares. Tras la localización, transcripción y vertimiento de los macrocurrículos encontrados a la base de datos (y la posterior depuración de esta muestra utilizando los criterios establecidos), se alcanzó un total de 935 materias o cursos.

El elemento más importante de la base de datos es la categorización de estos cursos según categorías generales o clases de materias, denominadas "áreas". Las propuestas de clasificación de las áreas que componen la Ciencia Política - lo que Duverger (DUVERGER, 1962, p. 545) llamaba su "dominio" han sido múltiples y casi tan antiguas como la disciplina misma. Sin embargo, uno de los primeros antecedentes de la época de la segunda posguerra es la "lista tipo" de la Organización de las Naciones Unidas para la Educación, la Ciencia y la Cultura (Unesco). Tras varias reuniones con expertos sobre el estatuto científico y epistemológico de la Ciencia Política en septiembre de 1948, la Unesco propuso un listado de tres secciones que componían la naciente disciplina y una serie de quince temas que componía las secciones ${ }^{8}$. Décadas después, cuando ya había

\footnotetext{
7 Por macrocurrículo (también llamado pensum, malla curricular o programa, según la universidad) se entiende el listado de materias o cursos que deben aprobar de forma obligatoria todos los estudiantes de un programa para acceder al título de politólogo o profesional en Ciencia Política de la institución en cuestión.

${ }^{8}$ En un documento clave para la historia de la Ciencia Política, la Unesco (1950) parecía olvidarse de la Teoría Política como sección o, como mínimo, incluía una cuarta: las Relaciones
} 
pasado el impulso funcionalista, el manual de Greenstein y Polsby (1975) en nueve volúmenes consideraban siete áreas sustantivas en la Ciencia Política. En tiempos más recientes, Goodin y Klingemann (1996) en el Nuevo Manual de Ciencia Política, y Goodin (2010) en la serie de manuales en diez volúmenes publicados por Oxford University Press, identificaron una serie de subdisciplinas o campos subdisciplinares. Por su parte, Cuéllar Argote (2007) identifica 17 disciplinas en el trabajo ya mencionado sobre la Ciencia Política en Colombia. Finalmente, el manual de Vallès (2008) propone sus propios campos temáticos, los cuales han sido reelaborados por Buitrago Pinedo et al. (2012) en un trabajo de investigación reciente. La Tabla 2 resume los contenidos de estas propuestas: 
Tabla 2 - División del campo disciplinar en áreas, según diversos autores

\section{Secciones (UNESCO, 1950)}

Teoría política; Instituciones políticas; Partidos, grupos y opinión pública; Relaciones Internacionales

\section{Campos (GREENSTEIN y POLSBY, 1975)}

Teoría política "micro"; Teoría política "macro"; Política no gubernamental; Instituciones y procesos gubernamentales; Políticas públicas y "policy-making"; Estrategias de investigación; Política internacional

\section{Subdisciplinas (GOODIN y KLINGEMANN, 1996)}

Instituciones políticas; Comportamiento político; Política comparada; Relaciones internacionales; Teoría política; Políticas públicas y administración pública; Economía política; Metodología política

\section{Disciplinas (CUÉLLAR ARGOTE, 2007)}

Histórica; Económica; Jurídica; Epistemológica-Metodológica; Relaciones

Internacionales; Administración Pública; Comunicación; Filosofía; Matemáticas;

Idiomas; Sociológica; Antropológica; Teológica; Actividad Física; Ética; Psicología;

\section{Otras}

\section{Campos temáticos (VALLÈS, 2008)}

Filosofía política; Teoría política; Política comparada; Instituciones públicas, administración pública y gobierno; Análisis del comportamiento político de individuos y grupos; Política exterior; Políticas públicas

\section{Campos subdisciplinares (GOODIN, 2010)}

Teoría política; Instituciones políticas; Derecho y política; Comportamiento político; Análisis de contexto; Política comparada; Relaciones internacionales; Economía política;

Políticas públicas; Metodología política

\section{Campos temáticos (BUITRAGO PINEDO et al., 2012)}

Filosofía política y teoría política; Instituciones públicas, administración pública y gobierno; Análisis del comportamiento político de individuos y grupos; Relaciones internacionales; Derechos humanos

Fuente: Elaboración propia con base en Unesco (1950), Greenstein y Polsby (1975), Goodin y Klingemann (1996), Cuéllar Argote (2007), Vallès (2008), Goodin (2010) y Buitrago Pinedo e. al. (2012).

Tras la revisión de propuestas, en ocasiones tan diversas, se construyó una clasificación propia de diez "áreas", más una categoría residual. Esta clasificación retoma puntos específicos de los referentes consultados: Se rechazó la unificación de la teoría política empírica y normativa que hacen Goodin y Klingemann (1996) y Goodin (2010), pero en vez de distinguir entre teoría "micro" y "macro" como hacen Greenstein y Polsby (1975), se adoptó una distinción entre teoría normativa y empírica, en línea con Vallès (2008). Se retoma de Cuéllar Argote (2007) y de Goodin (2010) la pluralidad de campos disciplinares o subdisciplinares que tienen el apellido "política". Finalmente, se abandonan áreas con más anclaje en el contexto anglosajón, como el de la conducta o comportamiento político. Así las 
cosas, la clasificación propuesta es la siguiente (cada área es seguida de tres ejemplos de materias, tomados de la base de datos):

1. Relaciones Internacionales y Política Internacional: "Teoría de las Relaciones Internacionales"; "Geopolítica"; "Política Exterior Colombiana".

2. Derecho Público y Derecho Político: "Derecho Constitucional"; "Derecho Internacional Público"; "Derechos Humanos y DIH".

3. Economía Política: "Economía Política"; "Economía Internacional"; "Principios de Economía".

4. Historia Política: "Historia de las Ideas Políticas"; "Historia de Colombia"; "Teoría de la Historia".

5. Sociología y Antropología políticas: "Acción Colectiva I: Movimientos Sociales y Políticos"; "Sociología Política"; "Conflicto y Paz en Colombia".

6. Metodología: "Análisis Cuantitativo"; "Diseño de Investigación"; "Epistemología de las Ciencias Sociales".

7. Comunicación Política y Lenguaje: "Análisis del Discurso"; "Mercadeo Político"; "Comunicación Política, Opinión Pública y Mercadeo".

8. Teoría política normativa: "Conceptos Políticos Fundamentales"; "Teoría y Filosofía Política"; "Teoría del Estado".

9. Teoría política empírica: "Introducción a la Ciencia Política"; "Política Comparada", "Sistema Político Colombiano".

10. Gobierno, Políticas Públicas y Administración Pública: "Teorías Organizacionales y Modelos de Gobierno"; "Administración Pública"; "Descentralización Política y Administración en Colombia".

11. Otras materias (categoría residual): "Ética y Bioética"; "Teología”; "Régimen Marítimo".

Utilizando esta clasificación y los datos recopilados, se puede empezar a aclarar el panorama en torno a la pregunta: ¿qué se enseña en los pregrados de Ciencia Política en el país? Para comenzar, y atendiendo a lo que cada pregrado dice de sí mismo, prácticamente ningún programa se declara disciplinar, lo cual en sí mismo es una primera tendencia a destacar. Por el contrario, hay una recurrente invocación a la inter, multi o trasdisciplinariedad y al diálogo de saberes, a la vez que hacen hincapié en la integralidad y flexibilidad de los macrocurrículos como una característica novedosa y que enriquece el campo politológico. Así, regularmente señalan un "diálogo de múltiples saberes de las ciencias sociales

\footnotetext{
${ }^{9}$ En adelante, su usarán los siguientes nombres o siglas para hacer referencia a estas áreas (principalmente, para simplificar la presentación de tablas y gráficos): Relaciones Internacionales y Política Internacional $=R R I I$; Derecho Público y Derecho Político $=$ Derecho , Economía Política = Economía $;$ Historia Política = Historia $;$ Sociología y Antropología Políticas = Sociología $;$ Metodología Política $=$ Métodos $;$ Comunicación Política y Lenguaje $=$ Comunicación $;$ Teoría Política Normativa $=$ TP Normativa $;$ Teoría Política Empírica $=$ TP Empírica ; Gobierno, Políticas Públicas y Administración Pública = Gobierno; y Otras materias = Otras .
} 
(...)" (Universidad de Antioquia), "una visión integral de la disciplina" (Universidad de los Andes), un "alto contenido multidisciplinar" (Universidad del Norte) y un "permanente diálogo con otros saberes" (Pontificia Universidad Javeriana, sede Cali), "pensar lo político desde lo múltiple, con un claro perfil humanístico" (Universidad EAFIT), entre otros ejemplos.

La Gráfica 2 ordena la distribución de los cursos ofrecidos según área estos datos de mayor a menor e ilustra de manera más clara que el área a la que se le dedica más espacio macrocurricular en el país es a la Metodología (15\% del total de materias analizadas), seguida de las Relaciones Internacionales (12,1\%), la Teoría Política Empírica (12\%) y el Derecho Político (10,7 \%). Si se unen ambas ramas de Teoría Política, conjugan una quinta parte de las materias ofrecidas en el país $(20,6 \%)$. En contraste, la Comunicación Política $(5,3 \%)$, la Sociología y Antropología Políticas $(6,8 \%)$ y la Historia Política $(7,7 \%)$ aparecen de forma más marginal en este panorama general.

Gráfica 2 - Proporción de materias enseñadas según área (\%)

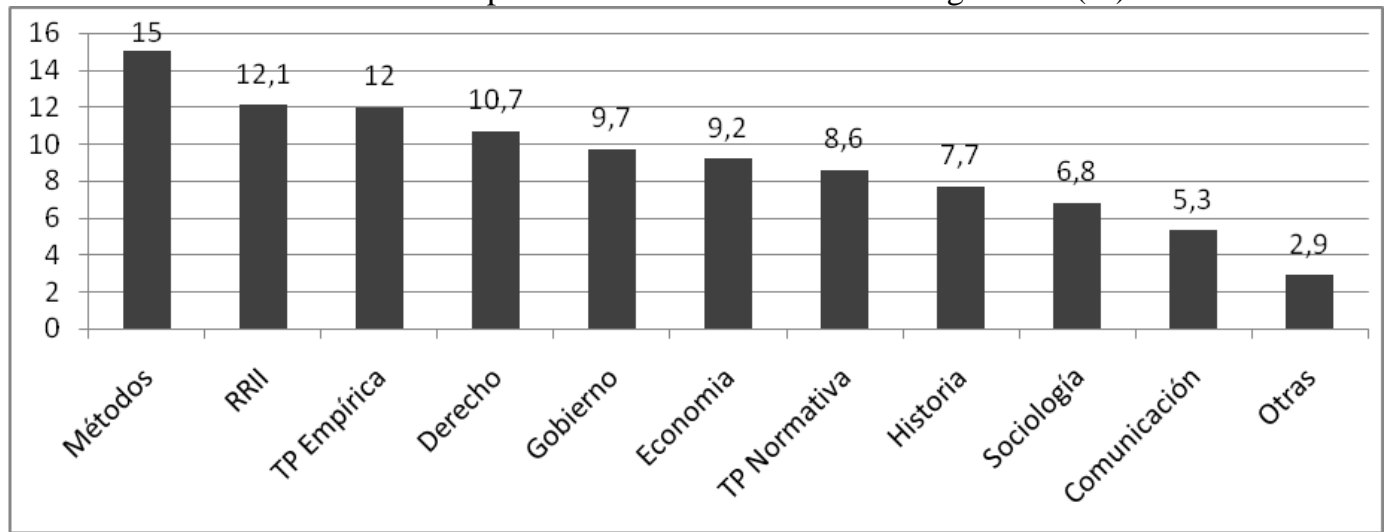

Fuente: Elaboración propia.

Con este panorama general en mente, se proponen tres comparaciones a manera de análisis macrocurricular: a) entre programas dictados en la ciudad de Bogotá y Cundinamarca por un lado, y aquellos que se imparten en otras regiones del país; b) entre programas de Ciencia o Ciencias Políticas y aquellos que toman otras denominaciones que aluden al Gobierno, las Relaciones Internacionales y la Resolución de Conflictos (disciplinas afines); y c) entre programas individuales, de forma tal que se puedan encontrar regularidades y diferencias, con especial énfasis en identificar programas "especializados" (aquellos que apuestan con fuerza a unas pocas áreas) y programas "amplios" (que apuestan a una cantidad amplia de áreas).

La Tabla Tabla 3 muestra la diferencia en la distribución de las materias dictadas en Bogotá y Cundinamarca, en comparación con el resto de las regiones del país. Vale la pena destacar aquellos casos en los que las diferencias son altas. Así, parece que en Bogotá se hace un énfasis un poco mayor en la Comunicación, 
las Relaciones Internacionales y los cursos de Gobierno y Políticas Públicas. Mientras, en otros lugares del país se enfatiza la enseñanza en Teoría Normativa y Metodología para la Ciencia Política. Esto es importante pues demuestra que, si bien poco importante, sí existe una diferencia en la manera en que se enseña la disciplina según donde se enseñe. El peso que tienen en Bogotá la Comunicación, las Relaciones Internacionales y el Gobierno puede obedecer a que es el centro político y mediático del país; por fuera de la capital y su área de directa influencia, el proceso de diferenciación institucional de la Ciencia Política con respecto a otras disciplinas, especialmente la Filosofía, puede ayudar a explicar la importancia de la formación en Teoría Normativa.

Ahondando en los hallazgos, se encuentra que existe un total de 11 cursos en cuyo nombre están incluidas las palabras "regional", "local" o ambas. De estas, solamente la asignatura "Gobierno Local y Regional" en la Universidad del Rosario se imparte en Bogotá; el resto se encuentran en las universidades EAFIT, Nacional de Colombia (sede Medellín), del Área Andina, Autónoma de Manizales, de Ibagué, del Tolima e ICESI. Quizás esto se relacione con algunas de las apuestas de programas ubicados por fuera de Bogotá, los cuales, en su mayoría, señalan que buscan contribuir al estudio de la política desde la región, atendiendo a los problemas locales-regionales y su articulación con lo nacional e internacional. Así, algunos están abocados al "análisis de los problemas de la región" (Universidad ICESI); otros postulan que "[...] el Programa se encuentra comprometido con el desarrollo regional [...] lograr una articulación de la Ciencia Política y la actividad política que permitan un desarrollo democrático en la región" (Universidad de Ibagué); o buscan "incidir de manera directa [...] en la Costa" (Universidad del Norte). Lo anterior tiene cierta relación con el proceso de descentralización política que el país vivió a partir de 1991 y que explica, en parte, la explosión de programas finalizando el siglo XX por fuera de la capital. Este proceso podría tener un impacto equiparable al de la creación de programas, en la conformación de los macrocurrículos. 
Tabla 3 - Comparación Bogotá y regiones (\%)

\begin{tabular}{c|c|c|c|c}
\hline Áreas & Bogotá & Otras regiones & Todos & Diferencia \\
\hline Comunicación & 7,6 & 3,3 & 5,3 & 4,4 \\
\hline RRII & 13,9 & 10,4 & 12,1 & 3,5 \\
\hline Gobierno & 11,2 & 8,4 & 9,7 & 2,8 \\
\hline Derecho & 11,2 & 10,2 & 10,7 & 1,0 \\
\hline Economía & 9,0 & 9,4 & 9,2 & $-0,4$ \\
\hline TP Empírica & 11,7 & 12,3 & 12,0 & $-0,6$ \\
\hline Historia & 7,4 & 8,0 & 7,7 & $-0,6$ \\
\hline Sociología & 6,3 & 7,4 & 6,8 & $-1,1$ \\
\hline Otras & 2,2 & 3,5 & 2,9 & $-1,2$ \\
\hline Métodos & 13,5 & 16,4 & 15,0 & $-2,9$ \\
\hline TP Normativa & 6,1 & 10,8 & 8,6 & $-4,8$ \\
\hline
\end{tabular}

Fuente: Elaboración propia.

En segundo lugar, la Tabla Tabla 4 muestra cómo se comporta la distribución de cursos según áreas en aquellos pregrados que se nombran a sí mismos de manera más "disciplinar" (Ciencia o Ciencias Políticas, por ejemplo) y aquellos que le agregan otros descriptores (como el de Gobierno o Relaciones Internacionales). No es de extrañar, pues, que en los primeros pesen mucho más las áreas de Teoría Política (ambas) y de Metodología ${ }^{10}$, a la vez que los otros pregrados le apuesten a la Economía, el Derecho y las Relaciones Internacionales. Esto indica que las denominaciones de los programas no son simples rótulos, sino que en parte expresan una apuesta disciplinar particular que se concreta en la elaboración y enseñanza de un macrocurrículo con sus propias características y énfasis en lo propiamente disciplinar o en lo multidisciplinar.

\footnotetext{
${ }^{10}$ El importante peso de la Comunicación Política en los pregrados más "disciplinares" es difícil de explicar, si bien puede tener una relación directa el enfoque de comunicación, marketing político y análisis de opinión pública, entendiéndolos como enfoques de la comunicación que han encontrado en la Ciencia Política un grado importante de énfasis disciplinar, esta categoría agrupa además asuntos afines a los estudios del lenguaje que no podría decirse, en estricto sentido, que forman parte de la estructura disciplinar de la Ciencia Política, como sí lo hacen de la Comunicación.
} 
Tabla 4 - Comparación entre programas con y sin descriptores adicionales (\%)

\begin{tabular}{c|c|c|c|c}
\hline Áreas & Total & Sin descriptores & Con descriptores & Diferencia \\
\hline TP Normativa & 8,6 & 9,8 & 5,9 & 3,9 \\
\hline Comunicación & 5,3 & 6,5 & 3,0 & 3,5 \\
\hline TP Empírica & 12,0 & 13,0 & 9,9 & 3,1 \\
\hline Métodos & 15,0 & 15,8 & 13,2 & 2,6 \\
\hline Sociología & 6,8 & 7,6 & 5,3 & 2,3 \\
\hline Historia & 7,7 & 7,6 & 7,9 & $-0,3$ \\
\hline Otras & 2,9 & 2,8 & 3,0 & $-0,1$ \\
\hline Gobierno & 9,7 & 9,5 & 10,2 & $-0,7$ \\
\hline RRII & 12,1 & 11,1 & 14,2 & $-3,1$ \\
\hline Derecho & 10,7 & 9,3 & 13,5 & $-4,2$ \\
\hline Economía & 9,2 & 7,0 & 13,9 & $-6,9$ \\
\hline
\end{tabular}

Fuente: Elaboración propia.

Finalmente, la Tabla 5 retoma esta idea de programas diferenciados e intenta darle un piso estadístico mucho más preciso a partir de los datos recopilados, comparando ya no con base en categorías amplias (Bogotá vs. regiones), sino desagregando programa por programa. En la 5 se muestran las distintas "apuestas macrocurriculares" de los 28 programas de pregrado analizados, en términos de las áreas curriculares a las que le otorgan mayor peso (este peso es indicado con una serie de asteriscos). Por ejemplo, el programa en Ciencia Política y Gobierno de la Universidad Jorge Tadeo Lozano dedica entre 10 y $15 \%$ de sus cursos a las áreas de Derecho (14,7\%), Metodología $(11,8 \%)$ y Gobierno (14,7\%), mientras que le dedica más del $20 \%$ al área de Teoría Política Empírica (20,6\%).

El análisis de esta tabla permite señalar dos cuestiones. Primero, distinguir entre dos tipos (laxamente definidos) de programas. Por un lado, aquellos que intentan abarcar un mayor campo dentro de las distintas áreas de la Ciencia Política ( $\geq 10 \%$ en múltiples áreas). Es el caso de los programas de la Universidad de la Sabana (8 de 11), las dos sedes de la Pontificia Universidad Javeriana (6 de 11), la Universidad Autónoma de Manizales (6 de 11) y la Universidad Sergio Arboleda (6 de 11). Por otro lado, se encuentran programas que enfatizan determinadas áreas ( $\geq 10 \%$ en pocas áreas $\mathrm{y} \geq 20 \%$ en por lo menos dos), como son los de la Universidad San Buenaventura, sede Cali (Relaciones Internacionales, Derecho y Economía), la Universidad del Rosario (Relaciones Internacionales y Gobierno) y la Universidad de los Andes (Metodología y Teoría Empírica).

La segunda cuestión que permite discernir este análisis es cuáles son las áreas de la Ciencia Política en Colombia en las que "todos juegan", esto es, aquellas en las que buena parte de los pregrados ha abarcado más del $10 \%$ de sus 
macrocurrículos. De las diez áreas sustanciales, solo Metodología (23 de 28 programas), Teoría Política Empírica (18 de 28) y Relaciones Internacionales (17 de 28) aparecen con fuerza en más de la mitad de los pregrados. A la inversa, también es de interés entender cuáles son aquellas áreas a las que pocos programas les apuestan (menos de 10). Como es de esperar, pocos programas le apuestan a la categoría residual (1 de 28); le siguen Comunicación Política (5 de 28), Sociología y Antropología Políticas (7 de 28) y Economía Política (8 de 28). De esta manera, se configura una suerte de "núcleo" disciplinar en torno al cual convergen buena parte de los programas: la formación metodológica e investigativa, la teórica y en política internacional. Mientras tanto, aquellas áreas que beben más de otras ciencias sociales y humanas tienen una presencia curricular menor en la medida en que no parece existir un consenso en torno a ellas, como parte indispensable de la formación politólogica. 
Tabla 5 - Énfasis curriculares de 28 pregrados según número de cursos por área

\begin{tabular}{|c|c|c|c|c|c|c|c|c|c|c|c|}
\hline Universidad & RRII & $\begin{array}{c}\text { Dere- } \\
\text { cho }\end{array}$ & $\begin{array}{c}\text { Econo- } \\
\text { mía }\end{array}$ & $\begin{array}{c}\text { Histo- } \\
\text { ria }\end{array}$ & \begin{tabular}{|c|} 
Socio- \\
logía
\end{tabular} & $\begin{array}{c}\text { Méto- } \\
\text { dos }\end{array}$ & $\begin{array}{c}\text { Comuni- } \\
\text { cación }\end{array}$ & $\begin{array}{c}\text { TP } \\
\text { Norm. }\end{array}$ & $\begin{array}{c}\text { TP } \\
\text { Emp. }\end{array}$ & $\begin{array}{c}\text { Gobier- } \\
\text { no }\end{array}$ & Otras \\
\hline $\begin{array}{c}\text { U. del } \\
\text { Rosario }\end{array}$ & $* * *$ & & & & & & & $*$ & & $* * *$ & \\
\hline $\begin{array}{c}\text { U. Tadeo } \\
\text { Lozano }\end{array}$ & & $*$ & & & & $*$ & & & $* * *$ & $*$ & \\
\hline U. del Norte & & & & $*$ & & $* *$ & & $*$ & $* *$ & & \\
\hline $\begin{array}{c}\text { Cervantina } \\
\text { San Agustín }\end{array}$ & $*$ & & $*$ & & $*$ & $*$ & $* *$ & & & & \\
\hline $\begin{array}{l}\text { U. del Área } \\
\text { Andina }\end{array}$ & & $*$ & & & $* *$ & $* *$ & & & & & \\
\hline $\begin{array}{l}\text { Poli. Granco- } \\
\text { lombiano }\end{array}$ & $* *$ & & & & & $* *$ & $* * *$ & & * & & \\
\hline PUJ - Bogotá & $*$ & & & & $*$ & $*$ & & $*$ & $*$ & $*$ & \\
\hline PUJ - Cali & & & & $*$ & $*$ & $* * *$ & & $*$ & $* *$ & & $*$ \\
\hline $\begin{array}{c}\text { Aut. de } \\
\text { Manizales }\end{array}$ & & $*$ & $*$ & $*$ & $*$ & $*$ & & & & $* * *$ & \\
\hline $\begin{array}{c}\text { U. de } \\
\text { Antioquia }\end{array}$ & & & & & & $* * *$ & & $*$ & $* *$ & & \\
\hline U. de Ibagué & & * & & & & $* * *$ & & $* *$ & $*$ & & \\
\hline $\begin{array}{l}\text { U. de La } \\
\text { Sabana }\end{array}$ & $*$ & $*$ & & $*$ & & $*$ & $*$ & $*$ & * & $*$ & \\
\hline $\begin{array}{c}\text { U. de los } \\
\text { Andes }\end{array}$ & & & & $*$ & & $* * *$ & & & $* * *$ & & \\
\hline $\begin{array}{c}\text { S. } \\
\text { Buenaventura } \\
- \text { Bog. }\end{array}$ & $* * *$ & $* * *$ & & & $*$ & & & & & $* *$ & \\
\hline $\begin{array}{c}\text { S. } \\
\text { Buenaventura } \\
\text { - Cali } \\
\end{array}$ & $* * *$ & $* * *$ & $* * *$ & & & & & & & $*$ & \\
\hline U. del Cauca & & & $*$ & $* *$ & & $* *$ & & & $* * *$ & & \\
\hline U. del Tolima & $*$ & & & & & $* * *$ & & $*$ & * & & \\
\hline U. EAFIT & $*$ & & $*$ & & & & $*$ & $* *$ & & & \\
\hline U. Externado & $*$ & $* *$ & $* * *$ & & & $* *$ & & & & $*$ & \\
\hline U. ICESI & & & & & & $* * *$ & & $*$ & $*$ & & \\
\hline $\begin{array}{c}\text { Militar Nueva } \\
\text { Granada }\end{array}$ & $* *$ & $* *$ & $* *$ & & & $* *$ & & & $* * *$ & & \\
\hline U. Mariana & $*$ & & & & & $* * *$ & & & $*$ & $*$ & \\
\hline UN - Bogotá & & & & $* *$ & & $* *$ & & $*$ & $*$ & & \\
\hline $\begin{array}{c}\text { UN- } \\
\text { Medellín }\end{array}$ & $* *$ & & & & & $*$ & & $* * *$ & $*$ & $*$ & \\
\hline $\begin{array}{c}\text { U. Pontif. } \\
\text { Bolivariana }\end{array}$ & $*$ & $* * *$ & & $*$ & & & & & $*$ & & \\
\hline $\begin{array}{l}\text { U. Santo } \\
\text { Tomás }\end{array}$ & $* * *$ & $*$ & & & & $*$ & $* *$ & & * & & \\
\hline $\begin{array}{l}\text { U. Sergio } \\
\text { Arboleda }\end{array}$ & $* *$ & $*$ & $*$ & $*$ & & $*$ & & & & $* *$ & \\
\hline $\begin{array}{c}\text { Tecnológica } \\
\text { de Bolívar }\end{array}$ & $* *$ & & & $* *$ & $*$ & $* *$ & & & & & \\
\hline
\end{tabular}

Nota: $*=$ de $10 \%$ a $14,99 \% ; * *=$ de $15 \%$ a $19,99 \%$; *** $=$ de $20 \%$ en adelante.

Fuente: Elaboración propia. 


\section{Conclusiones}

Analizar el estado de la Ciencia Política en el plano internacional, pero también en planos nacionales como el colombiano, implica optar por una consideración mixta entre estado de la disciplina e historias de la disciplina. Como se pudo ver a lo largo del presente trabajo, existen dos grandes perspectivas en el análisis del estado actual de la disciplina de la Ciencia Política: la perspectiva optimista o "whig" y la perspectiva escéptica o crítica; ambas se reflejan en el plano internacional, regional y nacional, con diversos matices y particularidades propias de cada entorno. El debate en cuanto a método(s), objeto(s) y enfoque(s), que aún persiste en la disciplina, lejos de pensarse como un obstáculo, puede verse como aquello que la mantiene viva y en constante transformación.

En el país, la historia de la disciplina tendría su punto de origen con la profesionalización lenta de las ciencias sociales en general a partir de los años cincuenta del siglo XX; continuaría en 1968 con el establecimiento, en la Universidad de los Andes, de la primera carrera de Ciencia Política; pasaría por la ampliación del abanico de temas tratados por politólogos y estudiosos de la política en las tres décadas siguientes; y desembocaría en una expansión inusitada de programas de formación profesional, tanto de pregrado como de posgrado, en Ciencia Política, con la particularidad de su expansión por fuera de la órbita de la capital, haciendo fuerte presencia en las regiones.

Mientras que las visiones optimistas, en general, celebran el crecimiento de la disciplina en el país tras un largo y trabajoso proceso, las perspectivas críticas se enfrentan respecto a si la Ciencia Política colombiana se ha ido "americanizando" o no, y sobre si esto es positivo o no. A esto habría que sumarle las discusiones de vieja data sobre el objeto de estudio, los métodos e incluso la denominación (ciencia, ciencias o estudios) de la disciplina, sobre las cuales hay múltiples posiciones. Esto indica que el desarrollo "positivo" de la misma dista de ser una "verdad revelada" y que el espacio de disenso sobre los logros y perspectivas de los politólogos en el país está aún vigente.

Es menester reiterar que este trabajo no presenta (ni pretendió presentar) una imagen total del estado de la formación universitaria de pregrado en Ciencia Política en Colombia. Se trata, más bien, de una visión parcial, centrada en aquello que se imparte en los distintos programas desde el nivel macrocurricular.

El análisis de casi mil materias de Ciencia Política y su clasificación según las distintas áreas que componen la disciplina permiten acercarse también a una conclusión preliminar en este aspecto. Existen hoy apuestas diferenciadas en por lo menos tres dimensiones. Primero, los pregrados ubicados en Bogotá son marginalmente más orientados hacia el Gobierno, la Comunicación y las Relaciones Internacionales, mientras que los regionales le apuestan más a la Teoría Política Normativa, posiblemente un legado de sus orígenes más recientes e interdisciplinares, que ha hecho también que los programas regionales adopten como objetivos la investigación en torno a sus contextos particulares y zonas de 
influencia. Segundo, es posible afirmar que "el nombre importa": agregar "Gobierno", "Relaciones Internacionales" o palabras similares a la denominación de un pregrado se relaciona con el grado de "disciplinariedad" de su apuesta macrocurricular. Tercero, mientras que por un lado existen programas que se inclinan por un mayor número de áreas (hasta 8 de 11), por el otro, hay un grupo de pregrados que prefieren enfatizar y concentrarse en unas pocas, a manera de sello distintivo.

El análisis estadístico presentado mostró tendencias importantes en torno a un "núcleo" disciplinar en el que convergen buena parte de los programas: la formación metodológica e investigativa, la teórica y en política internacional. Por otra parte, hay un núcleo de áreas ligadas a otras ciencias sociales y humanas, con una presencia curricular menos significativa, explicada en la falta de consenso en torno a ellas como parte indispensable de la formación politológica: Comunicación Política, Sociología y Antropología Políticas, y Economía Política.

Sin embargo, en los últimos años, la dinámica de la acreditación de alta calidad supone una mayor presión para empezar a tener estándares comunes y para que los programas se pregunten por los contenidos mínimos que deben cubrir para poder cumplir con la denominación que prometen. Igualmente, es posible vislumbrar que en un futuro la ACCPOL pueda jugar un papel más relevante en retroalimentar diseños curriculares y apoyar el proceso de reforma en los programas universitarios.

De cualquier manera, las posibles consecuencias - de una pluralidad de pregrados diferenciados según región, nombre y apuesta propia - merecen ser evaluadas, en tanto que esa expansión puede ser leída como el logro de cierta legitimidad académica y profesional de la Ciencia Política en el país o como un indicador de una institucionalización limitada que deriva en fragmentación y dispersión disciplinar. Para avanzar en esa línea habría que adicionar algunas preguntas: ¿hay diálogo y colaboración académica entre los programas?, ¿responden a procesos de estandarización profesional y a la presencia de una masa crítica de politólogos formados, con experiencia profesional e investigativa?, ¿se podría llegar a acuerdos por áreas específicas? Finalmente, de cara a los hallazgos previamente presentados, cabe plantearse el papel que tiene hoy la ACCPOL respecto a los procesos de institucionalización disciplinar, estandarización académica, cooperación y trabajo en red entre programas e inserción internacional.

Santiago Leyva Botero é Professor do Departamento de Humanidades da Universidade EAFIT e Chefe do Programa da Graduação em Ciências Políticas da Universidade EAFIT. E-mail: sleyvabo@eafit.edu.co 
María Fernanda Ramírez é Professora do Departamento de Humanidades da Universidade EAFIT e Coordenadora do Mestrado em Governo e Políticas Públicas da Universidade EAFIT. E-mail: mframire@eafit.edu.co

Patricia Muñoz Yi é Professora Associada da Faculdade de Ciência Política e Relações Internacionais da Pontifícia Universidade Javeriana e Diretora do Programa de Especialização em Opinião Pública e Mercado Político. Presidente da Associação Colombiana de Ciência Política (ACCPOL). E-mail: pmunoz@javeriana.edu.co

Andrés Preciado é Professor da Cátedra da Universidade EAFIT e Consultor do Centro de Análisis Político da Universidade EAFIT. Email: sleyvabo@eafit.edu.co

José Antonio Fortou é Professor da Escola de Ciências e Humanidades da Universidade EAFIT. Doutorando em Ciência Política da Ohio State University. E-mail: sleyvabo@eafit.edu.co

\section{Referencias}

ALMOND, Gabriel. Una disciplina segmentada: Escuelas y corrientes en las ciencias políticas. México D.F. Fondo de Cultura Económica, 1999.

ALONSO ESPINAL, Manoel. Los avances, los retos y la crisis de nuestro campo de conocimiento: Reflexiones oblicuas sobre el panorama de la Ciencia Política en Colombia. In: ROTH DEUBEL, Andre Noel; CÓRDOBA HOYOS, Lydia. La Ciencia Política en la Universidad del Cauca: Ponencias y Discursos de los Actos Conmemorativos, 1996-2001. Popayán: Editorial Universidad del Cauca, 2001. p. 55-85.

ALTMAN, David. From Fukuoka to Santiago: Institutionalization of Political Science in Latin America. PS: Political Science \& Politics, v. 39, n. 1, p. 196203, jan. 2006.

BeJARANO, Ana Maria; WILlS, María. La Ciencia Política en Colombia: de vocación a disciplina. Revista de Ciencia Política, Santiago, v. 25, n. 1, p. 111123, 2005.

BOTERO, Felipe. Cuatro décadas de estudios sobre partidos y elecciones. In: BOTERO, Felipe. Partidos y elecciones en Colombia. Bogotá: Editorial Universidad de los Andes, 2011. p. 19-40.

BUITRAGO PINEDO, Luis, CALLE OQUENDO, Stiven, Romero Rúa, Diego y Zapata Alzate, Stiven. Aproximación cuantitativa del desarrollo de la Ciencia Política en Colombia, 1968-2012: Un instrumento para el avance de la 
investigación. Trabajo de grado - Pregrado en Ciencia Política. Universidad de Antioquia, Medellín. 2012.

CANSINO, César. La muerte de la ciencia política. Buenos Aires: Editorial Sudamericana, 2008.

CHASQUETTI, Daniel. Construyendo instituciones: Examen de las asociaciones de Ciencia Política en el Cono Sur. In: LEYVA BOTERO, Santiago. La Ciencia Política en Colombia: ¿Una disciplina en institucionalización? Medellín: Universidad EAFIT, 2013. p. 287-308.

CUÉLLAR ARGOTE, Julián. Un diagnóstico a la enseñanza de la Ciencia Política en Colombia. Civilizar, Bogotá, v. 7, n. 13, p. 265-294, jul.-dez. 2007.

DUQUE DAZA, Javier. Tres momentos de la institucionalización de la enseñanza de la Ciencia Política en Colombia, 1968-2012. In: LEYVA BOTERO, Santiago. La Ciencia Política en Colombia: ¿Una disciplina en institucionalización? Medellín: Universidad EAFIT, 2013. p. 57-99.

DUVERGER, Maurice. Métodos de las ciencias sociales. Barcelona: Ariel, 1962.

FORTOU, José Antonio; LEYVA BOTERO, Santiago; PRECIADO, Andrés Felipe; RAMÍREZ, María Fernanda. Ciencia Política en Colombia: Una revisión de la literatura sobre el estado e historia de la disciplina en el país. In: LEYVA BOTERO, Santiago. La Ciencia Política en Colombia: ¿Una disciplina en institucionalización? Medellín: Universidad EAFIT, 2013. p. 2755.

GOODIN, Robert. The State of the Discipline, the Discipline of the State. In: GOODIN, Robert. The Oxford Handbook of Political Science. Oxford: Oxford University Press, 2010. p. 3-57.

GOODIN, Robert; KLINGEMANN, Hans-Dieter. Political Science: The Discipline. In: GOODIN, Robert; KLINGEMANN, Hans-Dieter. A New Handbook of Political Science. Oxford: Oxford University Press, 1996. p. 3132.

GREENSTEIN, Fred; POLSBY, Nelson. Handbook of Political Science. Reading: Addison-Wesley, 1975.

LEAL BUITRAGO, Francisco. La profesionalización de los estudios políticos en Colombia. Análisis Político, Bogotá, n. 3, jan.-abr. 1988.

LEAL BUITRAGO, Francisco. Prólogo: Cuatro décadas de Ciencia Política en la Universidad de los Andes. In: BOTERO, Felipe. Partidos y elecciones en Colombia. Bogotá: Editorial Universidad de los Andes, 2011. p. 8-18.

LOSADA, Rodrigo. Reflexiones sobre el estado actual de la Ciencia Política en Colombia. Papel Político, Bogotá, n. 16, p. 9-27, dez. 2004.

MURILLO CASTAÑO, Gabriel; UNGAR BLEIER, Elisabeth. Evolución y desarrollo de la Ciencia Política colombiana: Un proceso en marcha. Revista de Estudios Sociales, Bogotá, n. 9, p. 36-53, 1999. 
RESTREPO, Jorge Alberto; LÓPEZ, Sue Ellen; LÓPEZ, Laura; SÁNCHEZ, Luis Carlos. Ritmos de la producción discursiva en Análisis Político. Un análisis cuantitativo. Análisis político, Bogotá, v. 21, p. 81-89, 2008.

SÁNCHEZ DAVID, Rubén. Educación y Ciencia Política. In: ROTH DEUBEL, Andre Noel; CÓRDOBA HOYOS, Lydia. La Ciencia Política en la Universidad del Cauca: Ponencias y Discursos de los Actos Conmemorativos, 1996-2001. Popayán: Editorial Universidad del Cauca, 2001.

SÁNCHEZ, Rubén. El estudio de la Ciencia Política en Colombia. Bogotá: Editorial Universidad de los Andes, 1994.

SARTORI, Giovanni. ¿Hacia dónde va la ciencia política? Política y Gobierno, Santa Fé, v. XI, n. 2, p. 349-354, 2. sem. 2004.

UNESCO. Organización de las Naciones Unidas para la Educación, la Ciencia y la Cultura. Contemporary Political Science: A Survey of Methods, Research and Teaching. Lieja y París: UNESCO, 1950.

VALLÉS, Josep. Ciencia política: una introducción. Barcelona: Ariel, 2008.

Texto recebido em 18 de outubro 2013. Aprovado em 20 de novembro de 2013. 
